\title{
Modelling Mammographic Compression of the Breast
}

\author{
Jae-Hoon Chung, Vijay Rajagopal, Poul M.F. Nielsen, \\ and Martyn P. Nash \\ Auckland Bioengineering Institute, The University of Auckland, New Zealand \\ jh. chung@auckland.ac.nz
}

\begin{abstract}
We have developed a biomechanical model of the breast to simulate compression during mammographic imaging. The modelling framework was applied to a set of MR images of the breasts of a volunteer. Images of the uncompressed breast were segmented into skin and pectoral muscle, from which a finite element (FE) mesh of the left breast was generated using a nonlinear geometric fitting process. The compression plates within the breast MR coil were used to compress the volunteer's breasts by $32 \%$ in the latero-medial direction and the compressed breasts were subsequently imaged using MRI. The FE geometry of the uncompressed left breast was used to numerically simulate compression based on finite deformation elasticity coupled with contact mechanics, and individual-specific tissue properties. Accuracy of the simulated FE model was analysed by comparing the predicted surface data, and locations of three internal features within the compressed breast, with the equivalent experimental observations. Model predictions of the surface deformation yielded a RMS error of $1.5 \mathrm{~mm}$. The Euclidean errors in predicting the locations of three internal features were $4.1 \mathrm{~mm}, 4.1 \mathrm{~mm}$ and $6.5 \mathrm{~mm}$. Whilst the model reliably reproduced the compressive deformation, further investigations are required in order to test the validity of the underlying modelling assumptions. A reliable biomechanical model will provide a multi-modality imaging registration tool to help identify potential tumours observed between mammograms and other imaging modalities such as MRI or ultrasound.
\end{abstract}

\section{Introduction}

Mammography is currently the most common diagnostic and screening tool for breast cancers due to its relatively low cost and sensitivity in detecting carcinomas containing microcalcifications [1]. However, mammograms are $2 \mathrm{D}$ representations of a 3D object, and each mammogram is taken with the breast compressed along a different axis (typically mediolateral-oblique and cranio-caudal views are obtained). If a suspicious mass is identified on a mammogram, it is necessary to establish a spatial mapping of the mass between different views.

In order to account for the different deformations required to image mammograms, non-rigid registration techniques have traditionally been studied [2]. In recent years, biomechanical models of the breast using the finite element

D. Metaxas et al. (Eds.): MICCAI 2008, Part II, LNCS 5242, pp. 758 7652008.

(C) Springer-Verlag Berlin Heidelberg 2008 
(FE) method have received attention. However, previous biomechanical models have either used explicit displacement constraints to simulate compressions [34], boundary conditions that did not reflect the actual deformation of the breast (e.g. two-sided compression model to match one-sided compression in the experiments) 35], or validation studies were not adequately conducted to analyse the accuracy of the models 677.

In this study, we applied a FE modelling framework (tested using soft gel studies [89]) using breast MRI prior to, and after, mammographic-like compression. The main advantage of our modelling framework is that the model only requires the initial and final positions of the compression plates to simulate the compressive deformation using contact mechanics. The model can establish the spatial mapping between different mammograms, and also serves as a multimodality imaging registration tool to identify tumours seen in mammograms, MRI, and ultrasound. Such a multi-modality screening approach has shown to improve sensitivity and specificity [10] of breast cancer detection.

\section{Methods}

\subsection{Imaging}

A 1.5T Siemens MRI scanner (MAGNETOM Avanto System) was used for imaging the breast of a 36 year old volunteer. MRI was employed rather than a standard mammogram scanner, as MRI provides 3D volumetric data of the breast tissue structure. The volunteer was oriented in a prone position, with her breasts hanging under gravity in a breast coil. Axial MR images were obtained using a T2 weighted sequence with iPAT (integrated Parallel Acquisition Techniques), with a $350 \mathrm{~mm}$ by $350 \mathrm{~mm}$ field of view and a $2.5 \mathrm{~mm}$ slice thickness. Fig. 1 illustrates a MR image of the uncompressed breasts, with the skin and outer pectoral muscle surfaces segmented using CMGU1.

\section{$2.2 \quad$ Finite Element Mesh Generation}

A subset of the segmented data points for the left breast were selected as nodes of an initial FE mesh. Trilinear Lagrange finite elements were constructed from the selected nodes. 30 elements ( 6 elements in the medial-lateral $\xi_{1}$-direction, 5 elements in the inferior-superior $\xi_{2}$-direction, and a single element in the posterioranterior $\xi_{3}$-direction) were used to construct the initial mesh (Fig. 2, (a)). The trilinear mesh was then used to automatically construct and fit a mesh interpolated with smoothly continuous tricubic-Hermite basis functions. The nonlinear least-squares fitting process 11/2 involved minimisation of the Euclidean distance between the data points (of the skin and pectoral muscle layers) and their projections on the faces of the FE mesh. A root-mean-square (RMS) error of 0.69 $\mathrm{mm}$ was obtained between the fitted surface and the segmented surface data.

\footnotetext{
1 http://www.cmiss . org/cmgui
} 


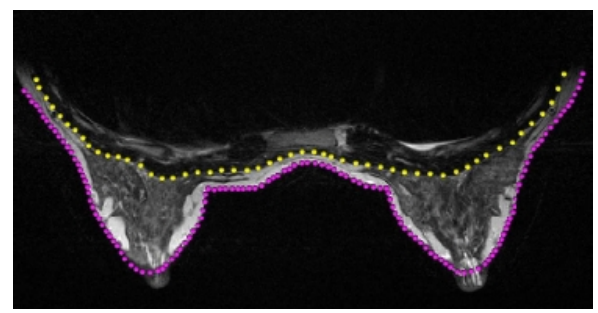

Fig. 1. A MR image of the uncompressed breasts. Segmented data for the skin (magenta) and pectoral muscle (yellow) surfaces are overlaid.

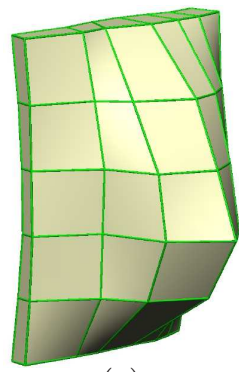

(a)

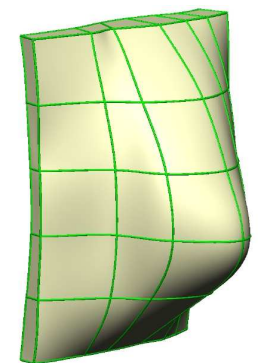

(b)

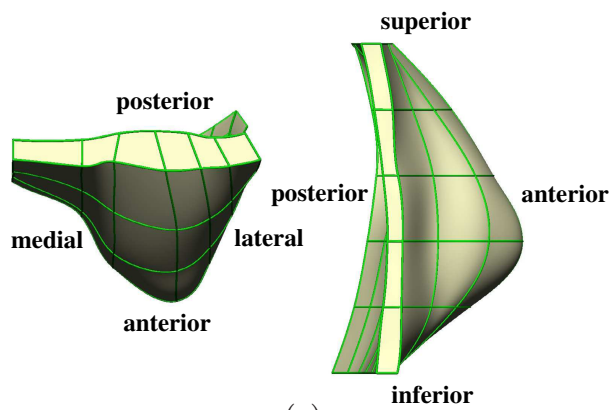

(c)

Fig. 2. Initial trilinear Lagrange mesh (a) and fitted tricubic-Hermite mesh of the uncompressed breast (b), (c)

This fitted model was then refined in order to ensure that the FE mesh consisted of sufficient geometric degrees of freedom to capture the compressive deformation. A refined model, with 231 nodes (5544 geometric degrees of freedom) and 120 elements ( 6 by 10 by 2), was used for simulating the mechanics due to compression.

\subsection{Modelling Compression}

The compression was modelled using the FE method applied to finite elasticity (in order to cope with the large deformation) coupled with contact mechanics to describe the interaction between the compression device and the breast. The reader is referred to 1314 for details. The FE model was analysed using CMISS2.

Based on a previous study using data from the same volunteer [15, a homogenous, incompressible and hyperelastic neo-Hookean constitutive relation (with the strain energy function defined as $\Psi=c_{1}\left(I_{1}-3\right)$, where $I_{1}$ is the $1^{\text {st }}$ invariant of the right Cauchy-Green strain tensor) was used to describe the mechanics of the breast tissue. The individual-specific material stiffness $\left(c_{1}\right)$ value of 0.1

\footnotetext{
2 http://www.cmiss.org
} 
$\mathrm{kPa}$ was estimated by matching the deformation of the breast from a neutrally buoyant reference configuration to the prone gravity-loaded state. Breast tissues adjacent to the pectoral muscle were constrained to be firmly attached to the muscle. The fitted FE model, shown in Fig. 2, was assumed to be in the unloaded reference state for the compressive mechanics problem.

The volunteer's breasts were compressed using the pads integrated in the breast coil. The pads at the medial sides of the breasts were immobile, whilst the lateral pads translated towards the medial pads, applying one-sided compression. The rounded edges of the lateral compression pad in the breast coil were represented using a tricubic-Hermite finite element mesh.

The width of the breast was reduced from $68.2 \mathrm{~mm}$ to $46.6 \mathrm{~mm}$, resulting in $32 \%$ compression. The compression was modelled using the penalty method [14] assuming frictionless contact, where the contact virtual work is defined as:

$$
\delta W_{c}=-\int_{S_{c}^{(1)}} t_{N} \mathbf{n} \cdot\left[\delta \mathbf{u}^{(1)}-\delta \mathbf{u}^{(2)}\right] d S_{c}^{(1)}
$$

where $\delta \mathbf{u}^{(1)} \& \delta \mathbf{u}^{(2)}$ are virtual displacements associated with the slave (breast) and the master (compression pads), respectively. $d S_{c}^{(1)}$ is the contact surface of the slave, and normal vectors $\mathbf{n}$ are defined on the master surface. The frictionless contact force $t_{N}$ is computed as

$$
t_{N}= \begin{cases}\epsilon_{N} g_{N} & \text { if } g_{N} \geq 0 \\ 0 & \text { otherwise }\end{cases}
$$

where $\epsilon_{N}$ is the penalty parameter, and $g_{N}$ is the gap function (penetration if positive). Details on the contact mechanics application to soft tissue mechanics can be found in 9 . A penalty value of $0.8 \mathrm{MPa} / \mathrm{m}$ was used in this study.

\section{Results}

Evaluation of modelling accuracy in predicting compressive deformations was conducted by comparing simulation results with experimental data derived from the MR images of the volunteer. As a measure of accuracy, surface deformations and the motion of three internal features were considered.

\subsection{Surface Deformation}

Surface data were segmented from the MR images of the compressed breast using CMGUI. These data were used to evaluate the modelling accuracy in predicting the surface deformations during compression. Fig. 3 illustrates the compressed model and the surface data of the volunteer's compressed breast segmented from the MR images, with the colour map showing the individual error magnitudes (in $\mathrm{mm}$ ) associated with each data point. The overall RMS error was $1.5 \mathrm{~mm}$. Note that only the surface data points corresponding to the compressed part of the breast were considered for analysing the geometric accuracy, since only the compressed tissues would appear in clinical mammograms. Localised surface deformation errors were observed at the edge of the compression pad, where the most abrupt breast tissue shape changes were located. 

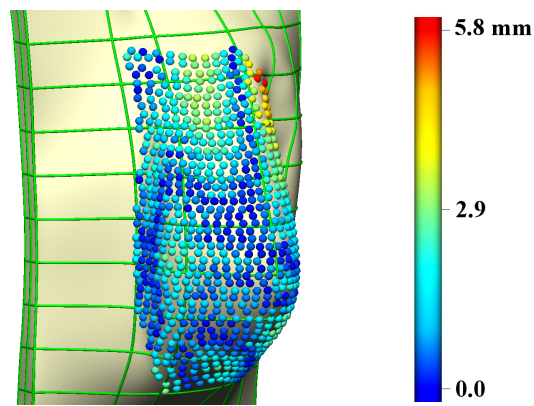

Fig. 3. Prediction of breast compression compared with the surface data obtained from MR image data. Surface data points are represented using a colour map indicating the individual error magnitudes (in $\mathrm{mm}$ ).

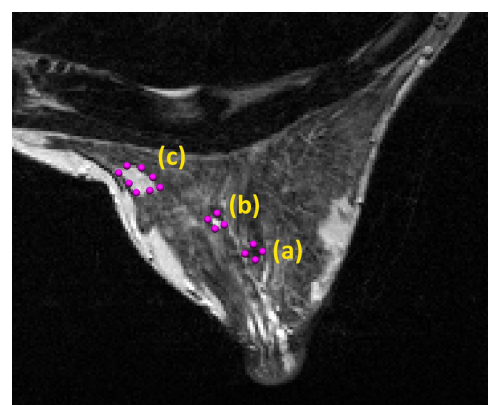

(a)

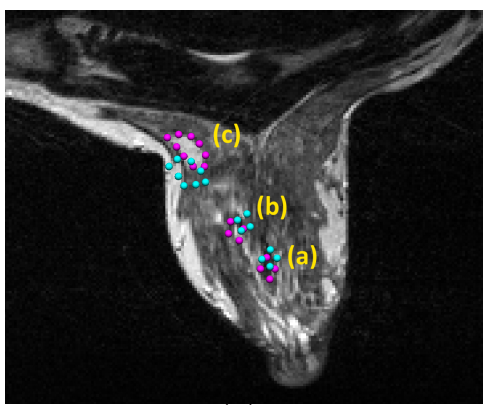

(b)

Fig. 4. (a) Segmented internal landmarks (magenta points) from the uncompressed MR image. (b) Predicted locations of 3 internal landmarks in the compressed state (cyan points) with experimental observations of the landmarks in the compressed MR image (magenta).

Table 1. Euclidean distances between experimental and predicted locations of 3 internal landmarks. The experimental displacements of the internal landmarks between uncompressed and compressed states are shown to illustrate the relative magnitudes of the errors.

\begin{tabular}{|c|c|c|c|}
\hline & (a) & (b) & (c) \\
\hline Euclidean error & $4.1 \mathrm{~mm}$ & $4.1 \mathrm{~mm}$ & $6.5 \mathrm{~mm}$ \\
\hline Displacement & $15.6 \mathrm{~mm}$ & $16.6 \mathrm{~mm}$ & $29.2 \mathrm{~mm}$ \\
\hline
\end{tabular}

\subsection{Internal Tissue Deformation}

From the MR images of the uncompressed breast, three identifiable internal features (landmarks) were segmented (Fig. 4. (a)). Based on the compression simulation result, the locations of these features in the compressed state were 


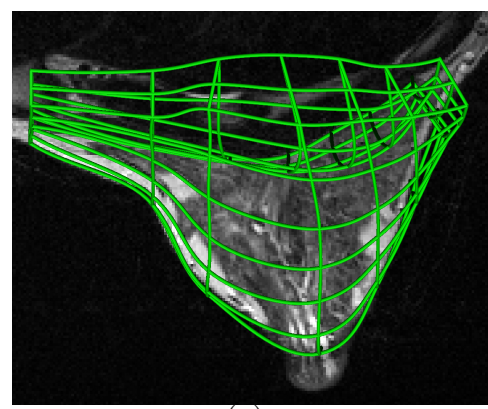

(a)

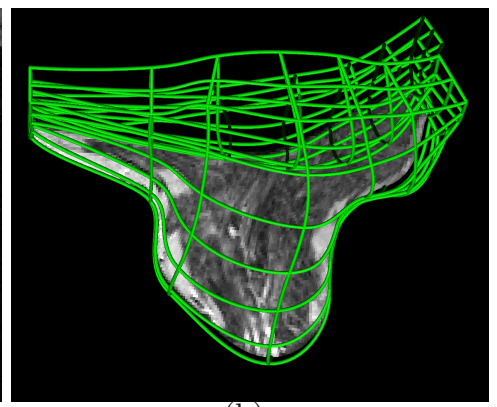

(b)

Fig. 5. (a) A MR image of the uncompressed breast embedded in the uncompressed FE model (green lines). (b) Warping of the uncompressed image in (a) using a biomechanical FE model of breast compression (green lines).

predicted and compared with data extracted from the MR images of the compressed breast, from which the locations of the same features were identified (Fig. 4. (b)). The centre of volume for each feature was calculated by averaging the coordinates of the segmented data points. Euclidean distances (errors) between the centre points of the predicted and experimental locations for the individual landmarks are shown in Table 1.

\subsection{Image Warping for Image Registration}

The predicted compressive deformation using the FE model can also be used to warp the original uncompressed MR image data. This warping was achieved by embedding the pixels locations of the uncompressed MR image (Fig.4, (a)) in the uncompressed breast model (Fig. 5. (a)), and then transforming the embedded pixels according to the deformation gradient tensor from the FE simulation (Fig. 5. (b)).

In future studies, these warped images may be used to more quantitatively validate the FE model by direct comparison with experimental MR images of the compressed breast using re-sampling and 3D image registration techniques. The example here demonstrates the potential use of the FE model for clinical breast image registration.

\section{Discussion}

This study illustrated the development of a biomechanical breast model, and its validation using MR images of a volunteer. The model was analysed using on our large deformation simulation framework, which has been tested and validated using experiments on soft gel phantoms. The volunteer's left breast was compressed by $32 \%$ and a corresponding FE model was constructed to simulate this deformation using contact mechanics. The accuracy of the compression simulation 
was evaluated by comparing experimental image data with the predicted surface deformations and the locations of 3 identifiable features. These preliminary results showed that the simplified model was able to capture the gross deformation under compression. It should be noted that the model only required positions of the compression pads before and after the compression (which are readily available in the clinical environment) to simulate the deformation using contact mechanics.

For improved modelling accuracy, we plan to further investigate the validity of the modelling assumptions made in this study. In particular, the heterogeneity of breast tissues (adipose and fibro-glandular tissues) and the fixed displacement condition of the breast tissue adjacent to the pectoral muscles will be scrutinised. The incorporation of the structural compartments, such as the skin and Cooper's ligaments, will also likely influence the mechanical behaviour of the compressive deformation. Numerical challenges associated with frictional contact mechanics in large deformation also need to be addressed in order to account for the interaction between the skin and compression pads, possibly by incorporating a recent mortar finite element approach [16]. In terms of evaluating the modelling accuracy, 3D image registration will be conducted between warped MR images from compression, and the experimental MR images of the compressed breast. A reliable biomechanical breast model will be useful as a robust and physically realistic registration tool for medical images of the breast and could potentially improve the sensitivity and/or specificity of breast cancer detection.

\section{Acknowledgements}

We gratefully acknowledge the financial support from Foundation for Research Science \& Technology and the University of Auckland Research Fund. We also thank Dr. Ralph Highnam for valuable discussions, and Sandra Winsor and Shelley Park at the Centre for Advanced MRI, Auckland, New Zealand, for their help in obtaining the MR images.

\section{References}

1. Sylvia, H.K., Dershaw, D., Schreer, I.: Diagnostic Breast Imaging: mammography, sonography, magnetic resonance imaging, and interventional procedures. 2 edn. Thieme, Stuttgart, New York (2001)

2. Rueckert, D., Sonoda, L., Hayes, D., Hill, D., Leach, M., Hawkes, D.: Nonrigid registration using free-form deformation: application to breast MR images. IEEE Transactions on Medical Imaging 18, 712-721 (1999)

3. Azar, F., Metaxas, D., Schnall, M.: Methods for modeling and predicting mechanical deformations of the breast under external perturbations. Medical Image Analysis 6(1), 1-27 (2002)

4. Tanner, C., Schnabel, J.A., Hill, D.L.G., Hawkes, D., Leach, M.O., Hose, D.R.: Factors influencing the accuracy of biomedical breast models. Medical Physics 33(6), 1758-1769 (2006) 
5. Ruiter, N.V.: Registration of X-ray Mammograms and MR-Volumes of the Female Breast based on Simulated Mammographic Deformation. PhD thesis, der Universitat Mannheim, Germany (2003)

6. Pathmanathan, P., Gavaghan, D., Whiteley, J., Brady, M., Nash, M.P., Nielsen, P.M., Rajagopal, V.: Predicting tumour location by simulating large deformations of the breast using a 3D finite element model and nonlinear elasticity. In: Barillot, C., Haynor, D.R., Hellier, P. (eds.) MICCAI 2004. LNCS, vol. 3217, pp. 217-224. Springer, Heidelberg (2004)

7. Samani, A., Bishop, J., Yaffe, M.J., Plewes, D.: Biomechanical 3-D finite element modeling of the human breast using MRI data. IEEE Transactions on Medical Imaging 20(4), 271-279 (2001)

8. Chung, J.H., Rajagopal, V., Nielsen, P.M., Nash, M.P.: A biomechanical model of mammographic compressions. Biomechanics and Modeling in Mechanobiology 7(1), 43-52 (2008)

9. Chung, J.H., Rajagopal, V., Laursen, T.A., Nielsen, P.M., Nash, M.P.: Frictional contact mechanics methods for soft materials: application to tracking breast cancers. Journal of Biomechanics 41(1), 69-77 (2008)

10. Malur, S., Wurdinger, S., Moritz, A., Michels, W., Schneider, A.: Comparison of written reports of mammography, sonography and magnetic resonance mammography for preoperative evaluation of breast lesions, with special emphasis on magnetic resonance mammography. Breast Cancer Research 3(1), 55-60 (2001)

11. Nielsen, P.M.: The Anatomy of the Heart: A Finite Element Model. PhD thesis, The University of Auckland, New Zealand (1987)

12. Bradley, C.P., Pullan, A.J., Hunter, P.J.: Geometric modeling of the human torso using cubic Hermite elements. Annals of Biomedical Engineering 25, 96-111 (1997)

13. Bonet, J., Wood, R.D.: Nonlinear Continuum Mechanics for Finite Element Analysis. Cambridge University Press, Cambridge (1997)

14. Laursen, T.A.: Computational Contact and Impact Mechanics. Springer, Berlin (2002)

15. Rajagopal, V., Lee, A., Chung, J.H., Warren, R., Highnam, R.P., Nielsen, P.M., Nash, M.P.: Towards tracking breast cancer across medical images using subjectspecific biomechanical models. In: Ayache, N., Ourselin, S., Maeder, A. (eds.) MICCAI 2007, Part I. LNCS, vol. 4791, pp. 651-658. Springer, Heidelberg (2007)

16. Puso, M.A., Laursen, T.A.: A mortar segment-to-segment contact method for large deformation solid mechanics. Computer Methods in Applied Mechanics and Engineering 193(6-8), 601-629 (2004) 